\title{
Variabilité Structurale Des Peuplements D’arbres En Forêt De Montagne Du Parc National De Kahuzi-Biega Et Ses Environs, RD. Congo
}

\author{
Imani Mugisho Gérard, Doctorant \\ Université Officielle de Bukavu, Faculté des Sciences et Sciences \\ Appliquées, Laboratoire d'Ecologie et Gestion \\ des Ressources Végétales, Bukavu, RDC \\ Université de Kisangani, Faculté de Sciences, \\ Laboratoire d’Ecologie et Aménagement, Kisangani
}

\section{Zapfack Louis, Professeur}

Université de Yaoundé 1, Département de Biologie et Physiologie Végétales, Laboratoire de Systématique et d’Ecologies Végétales, Yaoundé, Cameroun

\section{Bernard Riera, Professeur}

Museum National d'Histoire Naturelle, Laboratoire d'écologie générale, Brunoy, France

\section{Mwanga Mwanga Ithe Jean-Claude, Chercheur}

Centre de Recherche en Sciences Naturelles,

Département de Biologie, Laboratoire de Taxonomie Végétale

\section{Bulonvu Franclin, Assistant}

Institut Supérieur d'Agroforesterie et de Gestion de l'Environnement de

Kahuzi Biega, Département eaux et forêts, Bunyakiri, RDC

\section{Boyemba Faustin, Professeur}

Université de Kisangani, Faculté de Sciences, Laboratoire d'Ecologie et Aménagement, Kisangani

\section{doi: 10.19044/esj.2016.v12n23p88 URL:http://dx.doi.org/10.19044/esj.2016.v12n23p88}

\begin{abstract}
This work aims to determine the change in the diametric structure, density, basal area, height and dominance of trees $\mathrm{dbh} \geq 10 \mathrm{~cm}$ depending on altitude range or forest types. The study was conducted in 30 ha in Kahuzi Biega National Park and surrounding areas in DR Congo. In total, 16,797 individual trees were surveyed. The number of family, genus and species regress following the altitudinal gradient as the density increases. Mountain ecosystems in Kahuzi-Biega and its surroundings are dominated by Meliaceae, Euphorbiaceae, Moraceae, Fabaceae and Rubiaceae families. The Chi square analysis showed that the diametric structure of vegetation
\end{abstract}


succession following a progressive dynamic and varies according to altitude gradient. The average basal area was $35 \mathrm{~m}^{2} / \mathrm{ha}$. The analysis of variance (ANOVA) and post hoc Tukey test does not prove the impact of altitude on the basal area. But it becomes low in elevation due to the reduced diameter of the trees. The vertical structure was analyzed according to the classification IUFRO (International Union of Forest Research Organizations). The maximum height was $48 \mathrm{~m}$ and becomes lower in altitude. The tree height diameter relationship varies according altitude range so that the models H/D in this area should be developed for each forest type. We determine that the dominance of woody species following the altitude effect; only Strombosia scheffleri and Xymalos monospora dominated by its basal area, abundance and frequency of the various altitude horizons.

Keywords: Variability, structure, diameter, altitude range, density, basal area, Kahuzi-Biega and its surroundings

\section{Résumé}

Ce travail a pour but de déterminer le changement de la structure diamétrique, de la densité, de la surface terrière, de la hauteur et de la dominance des arbres à $\mathrm{dbh} \geq 10 \mathrm{~cm}$ selon les horizons d'altitude ou types forestiers. L'étude s'est effectuée dans 30 ha au Parc National de Kahuzi Biega et ses environs en RD Congo. Au total 16797 individus ont été inventoriés. Le nombre de famille, de genre et d'espèce régresse suivant le gradient altitudinal pendant que la densité augmente. Les écosystèmes montagneux au Parc National de Kahuzi-Biega et ses environs sont des formations à Meliaceae, Euphorbiaceae, Moraceae, Fabaceae et Rubiaceae. Le Chi carré a montré que la structure diamétrique de la végétation suit une succession progressive et varie selon les horizons d'altitude. La surface terrière moyenne est de $35 \mathrm{~m}^{2} / \mathrm{ha}$. L'analyse de la variance suivie d'un test post hoc de Tukey ne prouve pas l'influence de l'altitude sur la surface terrière. Mais celle-ci devient faible avec l'élévation en lien avec une diminution du diamètre des arbres. La structure verticale a été analysée suivant la classification IUFRO (International Union of Forest Research Organizations). La hauteur maximale est de $48 \mathrm{~m}$ et devient de plus en plus faible avec l'altitude. La relation hauteur diamètre des arbres varie selon les horizons d'altitude de sorte que les modèles $H / D$ dans cette région devaient être développés pour chaque type forestier. La dominance des espèces ligneuses suit l'effet d'altitude; seules Strombosia scheffleri et Xymalos monospora dominent par leur surface terrière, leur abondance et leur fréquence en relation avec les différents horizons d'altitude 
Mots clés : Structure diamétrique, altitude, densité, surface terrière, KahuziBiega

\section{Introduction}

La structure diamétrique des arbres, leur densité, leur surface terrière et leur hauteur sont influencées par les facteurs environnementaux, comme l'altitude, les pentes, la luminosité (Givnish, 1999 ; Lovett et al., 2006; Alves et al., 2010; Biresaw and Pavliš, 2010), par les types d'utilisation de terre ou les formations végétales (Lieberman et al., 1996; Zapfack et al., 2002; Ren et al., 2006; Bouko et al., 2007; Rahaingoson et al., 2013; Lee et al., 2014). Hormis ces facteurs clés, la structure d'un écosystème peut être, aussi influée par des facteurs naturels comme la présence ou l'absence de lianes envahissantes (Masumbuko et al., 2012), l'intensité de la perturbation de l'écosystème favorisant des trouées (Huang et al. 2003; Sahu et al., 2008; Boyemba, 2011), ou des vents violents (Masumbuko et al., 2012 ; Mangambu et al., 2015). De ces faits, l'analyse de la structure de la végétation et sa variabilité sont des indicateurs pour tester la théorie écologique du fonctionnement de l'écosystème (Ostertag et al., 2014) et comprendre sa dynamique (Bogaert et Mahamane, 2005). Le rôle de la séquestration de carbone par les forêts en dépend. Par exemple, pour certains auteurs comme Bastin et al., (2015), la fonction de séquestration de carbone à travers l'accumulation de la biomasse aérienne en forêt africaine peut être prédite avec précision à partir de quelques grands arbres, représentant 5\% d'arbres de la parcelle.

Ainsi, il est démontré que dans la région tropicale, la variabilité de la structure de la forêt peut être grande. Cette variabilité peut concerner les formations situées entre continent et dans un même continent (Phillips et al., 1994; Terborgh and Andresen, 1998; Ter Steege et al., 2000) mais également entre les différents sites d'une même forêt selon les conditions locales (Proctor et al., 1983). Toutefois, en forêt de montagne, les études comparatives de la variabilité structurale entre les types forestiers dans un même massif forestier sont rares et peu documentées (Fashing and Gathua 2004; Mangambu et al. 2015).

Néanmoins, les auteurs s’accordent au fait que dans ces formations montagnardes, la variabilité structurale pourrait être mesurée entre les sites sur base des conditions écologiques particulières locales (Lovett et al., 2006; Feldpausch et al., 2011). C'est ainsi que, les conditions climatiques, par exemple, influencent la composition et la structure de la végétation (Dale et al., 2000; Lasch et al., 2002). En zone de montagne, les espèces sont sensibles aux effets du changement climatique (Imani et al., 2016b). Lorsque le climat devient favorable, on note une expansion de la zone forestière aux 
altitudes inférieures et les conditions climatiques défavorables occasionnent un rétrécissement de la zone forestière (Kabonyi, 2012).

Dans le contexte du Parc National de Kahuzi Biega (PNKB) et ses environs, situés dans la partie sud de la chaîne de montagnes KivuRuwenzori, d'axe NNE-SSW (Nord Nord Est- Sud Sud Ouest), longeant à l'ouest, le rift Albertin (Mangambu, 2013), la forêt montagnarde est subdivisée en quatre types forestiers suivant le gradient d'altitude entre 1250 et $2600 \mathrm{~m}$ (Imani et al., 2016a). Il s'agit du type sub montagnard, du type montagnard horizon inférieur, du type montagnard horizon moyen et du type montagnard horizon supérieur. La composition floristique entre ces types est différente (Imani et al., 2016a). C'est ainsi que ce présent travail cherche à déterminer, d'une part, la variabilité de la structure diamétrique, de la densité, de la surface terrière et de la hauteur entre ces types forestiers et d'autre part à mettre en évidence les espèces et familles écologiquement importantes pour chacune de ces formations. L'étude a pour but de déterminer le changement dans la structure diamétrique, la densité, la surface terrière et la hauteur des arbres selon les tranches d'altitude ou types forestiers. Elle permettra de déterminer les espèces écologiquement importantes de chaque type forestier. On postule que la variabilité structurale (structure diamétrique, densité, surface terrière et hauteur) est forte entre les différentes communautés distinguées le long du gradient altitudinal.

\section{Materiel et methode \\ Zone d'étude}

En RD Congo, les forêts de montagne se situent à l'Est du pays, dans la zone du Rift Albertin (Pierlot, 1966). Cette étude a été effectuée au sein de la partie haute altitude du Parc National de Kahuzi-Biega et les forêts communautaires de Bunyakiri qui se trouvent dans les escarpements du mont Kahuzi (Runge, 2007) (Figure 1). Ces deux sites appartenaient au même massif et ne sont pas différents par leur composition floristique typique de forêt de montagne (Werger, 1973). La réserve du Kahuzi a été créée en 1937 puis érigée en Parc national de Kahuzi-Biega (PNKB) le 30 novembre 1970 sous la loi nº 70/31630/11/70 (Mangambu et al., 2010). 


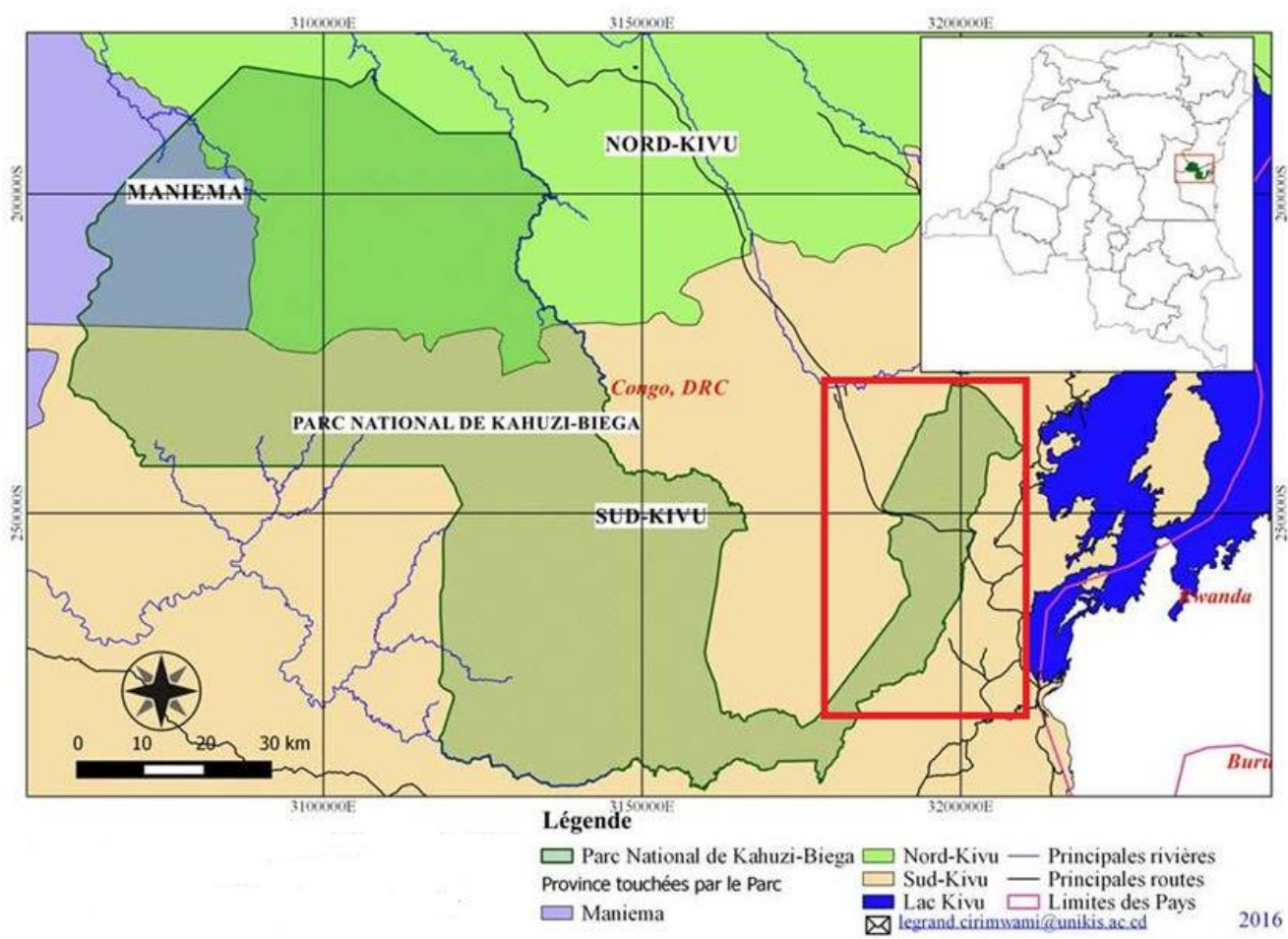

Figure 1: Localisation de la zone d'étude à l'Est de la RD Congo.

Les forêts de montagne se trouvent entre 1250 et $2600 \mathrm{~m}$ d'altitude. Au-delà de $2600 \mathrm{~m}$, commence la végétation afro-subalpin. Dans cette zone de montagne, la température moyenne est de $20,5^{\circ} \mathrm{C}$. Les précipitations sont de 1500- $2000 \mathrm{~mm}$ en moyenne et la radiation globale moyenne de 421,8 calories/cm3/mois (Yamagiwa et al., 2005). L'humidité relative moyenne est de 86 \% (Mangambu et al., 2013). Le sol au Kahuzi et dans les forêts communautaires est majoritairement sablo-argileux ou argileux avec un $\mathrm{pH}$ acide (données non publiées).

\section{Collecte de données}

Un échantillonnage de tous les individus ligneux d'arbres ayant un $\mathrm{dbh} \geq$ à $10 \mathrm{~cm}$ à $1,30 \mathrm{~m}$ a été effectué dans 30 parcelles de 1 ha chacune placées dans les différentes formations stables entre 1250 et $2600 \mathrm{~m}$ d'altitude (Wagner et al., 2010; Baraloto et al., 2013). Pour chaque individu, son diamètre a été mesuré à l'aide d'un dbh mètre, et sa hauteur a été prise avec un hypsomètre (Laser) sur quelques individus de chaque espèce par classe de diamètre à la parcelle (Feldpausch et al., 2011, 2012). Les identifications des espèces récoltées sur le terrain ont été vérifiées à la fois par comparaison avec les spécimens de référence conservés dans l’Herbarium du Centre des Recherches en Sciences Naturelles de Lwiro 
(LWI), de l'INERA Mulungu (MLGU) et dans celui du Jardin Botanique National de Belgique (BR).

\section{Analyse de données}

Les différents types forestiers dans la zone d'étude ont été pris en compte : Le type d'étage sub montagnard (1250-1500m), le type montagnard horizon inferieur (1500-1800m), type montagnard , horizon moyen (18002400m) et le type montagnard, horizon supérieur (2400-2600m).

Pour ce qui est de la structure de la végétation, 8 classes de diamètre ont été constituées sur base de données de dbh des espèces et suivant chaque type. Les classes sont de $10 \mathrm{~cm}$ d'intervalle, la première de $10-20 \mathrm{~cm}$ et la dernière $\geq 80 \mathrm{~cm}$. Afin de comparer la densité entre les différentes classes selon les types forestiers, les tests du Chi-carré $\left(\chi^{2}\right)$ d’indépendance ont été appliqués et la contribution des classes au test $\chi^{2}$ mise en évidence sur base de leurs effectifs.

La structure verticale a été analysée à l'aide du système de classification de l'IUFRO (International Union of Forest Research Organizations) tel que repris par Gurmessa et al., (2012). Ce système reconnait les horizons inférieur, moyen et supérieur d'une structure verticale forestière sur base de la hauteur maximale.

Pour déterminer les espèces les plus dominantes par type forestier, on a calculé l'indice de Valeur d'Importance (IVI) ou Importance Value Index (Curtis and Mclntosh, 1950) (Eq1) qui représente la somme, pour chaque espèce, de la Densité relative (Dr) et de la Dominance relative (Dor). Etant donné que les parcelles sont isolées, la fréquence relative n’est pas considérée. La valeur de l'IVI est en pourcentage et varie entre 0 à 200. Les dix premières espèces dominantes ont été considérées pour chaque type forestier.

$I V I_{(i)}=D r_{(i)}+\operatorname{Dor}_{(i)}$

Avec $\operatorname{Dr}(i)=\frac{N I}{N} * 100$ et $\operatorname{Dor}(i)=\frac{S T(i)}{S T(t)} * 100$

Où $\mathrm{N}=$ nombre total d'individus et $\mathrm{N}_{\mathrm{i}}=$ Nombre d'individus de l'espèce (i); $\mathrm{ST}_{(\mathrm{t})}=$ Surface terrière totale de la parcelle équivalant à :

$\mathrm{ST}$ totale $=\sum \mathrm{ST}_{(\mathrm{i})}$

Avec $\mathrm{ST}_{(\mathrm{i})}=$ surface terrière d'une espèce donnée.

$S T_{(i)}=\pi D^{2} / 4(\mathrm{D}=$ Diamètre en $\mathrm{m}) \quad \mathrm{Eq}(3)$

Pour tester les différences de hauteur, de surface terrière entre les types forestiers, on a réalisé les analyses de variance à un facteur (suivies de tests de Tukey HSD, Honest Significant Differences, pour comparer les moyennes lorsque cela était nécessaire). L’analyse de régression multiple a été faite pour déterminer la diminution du nombre de genre, de famille, d'espèce et d'individus avec l'augmentation d'altitude. 
Pour décrire les relations entre le diamètre et la hauteur, la surface terrière et la densité, des corrélations linéaires ont été réalisées. Tous les tests statistiques ont été réalisés avec le logiciel R 3.02. Le seuil de significativité étant de 5\%.

\section{Resultats}

\section{Variabilité de la densité et richesse spécifique}

On a recensé 16797 individus dans l'intervalle altitudinale entre 1250 et $2600 \mathrm{~m}$ dans 30 ha. Ces individus sont groupés en 212 espèces (morpho-taxa), 161 genres et 66 familles. Le nombre de famille, de genre, d'espèce et d'individus varie avec les différents types forestiers (tableau 1)

Tableau 1: Variation du nombre de famille, de genre, d'espèces et d'individus entre les

types forestiers

\begin{tabular}{|c|c|c|c|c|}
\hline Catégorie & $\begin{array}{c}\text { Type Sub- } \\
\text { montagnard } \\
\left(\begin{array}{c}1250-1500 \\
\mathrm{~m})\end{array}\right.\end{array}$ & $\begin{array}{c}\text { Type montagnard, } \\
\text { horizon inférieur } \\
(1500-1800 \mathrm{~m}\end{array}$ & $\begin{array}{c}\text { Type montagnard } \\
\text { horizon moyen } \\
(1800-2400 \mathrm{~m})\end{array}$ & $\begin{array}{c}\text { Type montagnard } \\
\text { horizon supérieur } \\
(2400-2600 \mathrm{~m})\end{array}$ \\
\hline Famille & 46 & 41 & 38 & 17 \\
\hline Genre & 107 & 82 & 72 & 25 \\
\hline Espèce & 124 & 100 & 91 & 28 \\
\hline Ni total & 2630 & 3067 & 7623 & 3477 \\
\hline $\begin{array}{c}\text { Ni moyen, } \\
\text { SD }\end{array}$ & $438 \pm 25$ & $511 \pm 55$ & $545 \pm 199$ & $869 \pm 478$ \\
\hline
\end{tabular}

Légende : $\mathrm{Ni}=$ Nombre d'individus SD = Ecart type

En effet, on note une régression du nombre de famille $\left(R^{2} 0,57, t\right.$ value $-6,12 ; p$ value $<0,001)$, de genre $\left(R^{2} 0,65, t\right.$ value $-7.27 ; p$ value $<0$, 001), du type sub-montagnard au type montagnard horizon supérieur. Seul le nombre d'individus augmente lorsqu'on se retrouve aux étages supérieurs de la forêt de montagne mais avec une forte variabilité pour les types montagnards horizon moyen et supérieur.

Les forêts sub-montagnardes sont des formations à Fabaceae (16 espèces), Euphorbiaceae (14 espèces), Moraceae (11 espèces) et Meliaceae (7 espèces). L'horizon montagnard inférieur est dominé par les Euphorbiaceae (12 espèces), Meliaceae (7 espèces), Moraceae (8 espèces), Clusiaceae (5 espèces). Pour l'horizon montagnard horizon moyen, ce sont les Euphorbiaceae ( 7 espèces), Fabaceae ( 7 espèces), Meliaceae ( 5 espèces) et enfin pour l'horizon montagnard supérieur, les Myrtaceae (3 espèces), Stilbaceae (2 espèces), Fabaceae (3 espèces), Ericaceae (2 espèces) dominent. Par ailleurs, dans chaque type forestier, le sous-bois est dominé par la famille des Rubiaceae sur base de la richesse spécifique et Monimiaceae sur base de l'abondance.

La figure 2 indique l'importance des familles selon leur abondance spécifique par tranche altitudinale. 


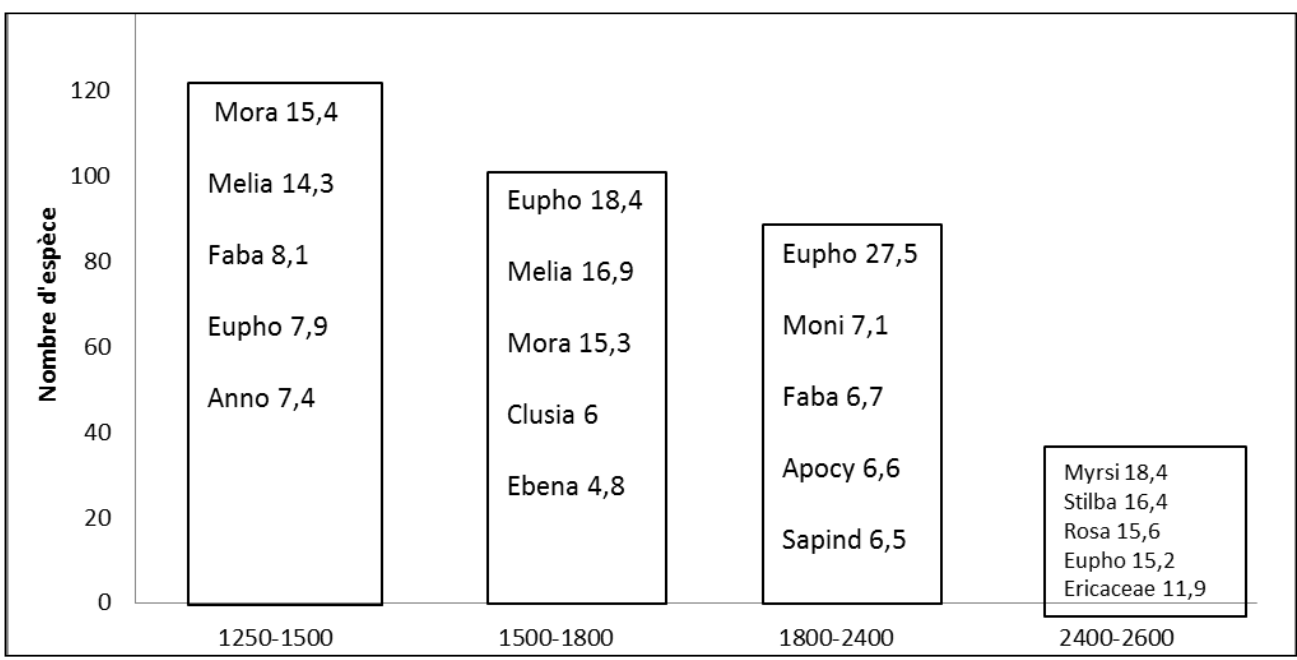

Figure 2: les cinq premières familles les mieux représentées dans les différents types forestiers (la valeur présentée est la densité relative). A l'échelle de l'axe, est indiquée la richesse spécifique suivant chaque tranche d'altitude. Mora= Moracaea, Melia =Meliaceae, Faba $=$ Fabaceae, Eupho=Euphorbiaceae, $A n n o=$ Annonaceae, Clusia= Clusiaceae, Ebena $=$ Ebenaceae, Moni $=$ Monimiaceae, Apocy $=$ Apocynaceae, Sapind $=$ Sapindaceae, Myrsi= Myrsinaceae, Stilba= Stilbaceae, Rosa= Rosaceae.

En effet, cette figure met en évidence le fait que les familles ayant plus d'espèces sont celles également dotées de nombreux individus. Cependant, les Euphorbiaceae et Monimiaceae ont, respectivement beaucoup d'individus et une seule espèce par tranche entre 1800-2400 et 2400-2600.

\section{Variabilité de la structure de la végétation}

\section{Structure diamétrique inter type forestier}

Pour mieux comparer la structure de la végétation et suivant le diamètre des arbres, 8 classes de diamètre ont été constituées. Sur l'ensemble de la communauté, la distribution suit une succession progressive avec une structure diamétrique en $\mathrm{J}$ inversé (Figure 3 ), typique des forêts tropicales non perturbées. La distribution diamétrique est variable $\left(\chi^{2}=621,9\right.$; $p<$ 0,001) (Figure 4). Les deux premières classes inférieures (de 10 à $30 \mathrm{~cm}$ ) ont plus d'individus que les classes supérieures (soit $66 \%$ au type submontagnard, $76 \%$ au montagnard horizon inférieur, $80 \%$ au montagnard horizon moyen et $90 \%$ au montagnard horizon supérieur). 


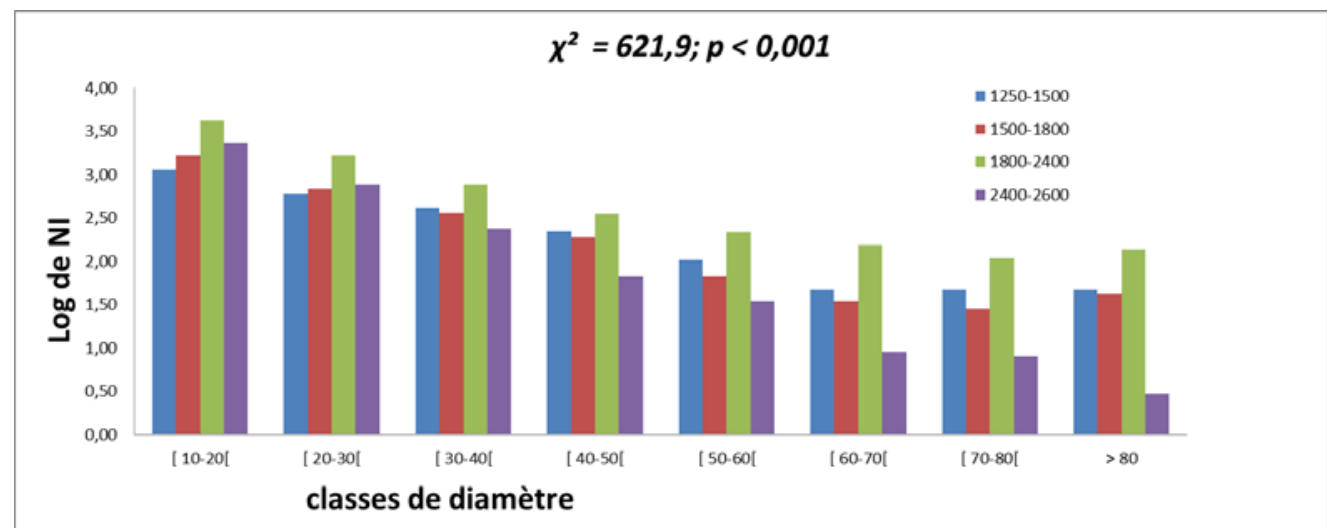

Figure 3 : Distribution diamétrique des tiges inventoriées suivant les types forestiers. Le nombre d'individus est transformé en logarithme. Type Sub-montagnard 1250-1500 m, Type montagnard horizon inférieur 1500-1800 m, Type montagnard horizon moyen 1800$2400 \mathrm{~m}$ et Type montagnard horizon supérieur 2400-2600 m.

L'analyse des contributions de chaque classe de diamètre à la statistique $\chi^{2}$ totale montre que, dans les classes $30-40 \mathrm{~cm}\left(\chi^{2} 9,4\right.$; résidus $7,7)$ et $40-50 \mathrm{~cm}\left(\chi^{2} 10,5\right.$; résidus 8,1$)$, l'effectif de la tranche $1250-1500$ a le poids le plus important, tout comme les effectifs des classes $60-70 \mathrm{~cm}\left(\chi^{2}\right.$ $2,7$; résidus 4,1$)$ de la tranche $1800-2400$ et $10-20 \mathrm{~cm}\left(\chi^{2} 13,8\right.$; résidus 9,3 ) de la tranche 2400-2600.

\section{Structure diamétrique intra type forestier}

Chaque type forestier est constitué d'un nombre donné de parcelles forestières qui n’ont pas forcement la même structure diamétrique. L'analyse de chi carré indique qu'il existe une variabilité de cette structure à l'intérieur même d'un type forestier. Les classes inférieures ont plus d'individus que les classes supérieures. En raison de la valeur du chi carré, on note que cette variabilité se présente différemment pour les types sub montagnard $\left(\chi^{2}=71,6\right.$ $; p<0,001)$ et montagnard horizon inférieur $\left(\chi^{2}=50,9 ; p=0,0016\right)$ comparer aux types montagnard horizon moyen $\left(\chi^{2}=855,4 ; p<0,001\right)$ et horizon supérieur $\left(\chi^{2}=139,8 ; p<0,001\right)$. La figure 4 résume cette situation : 


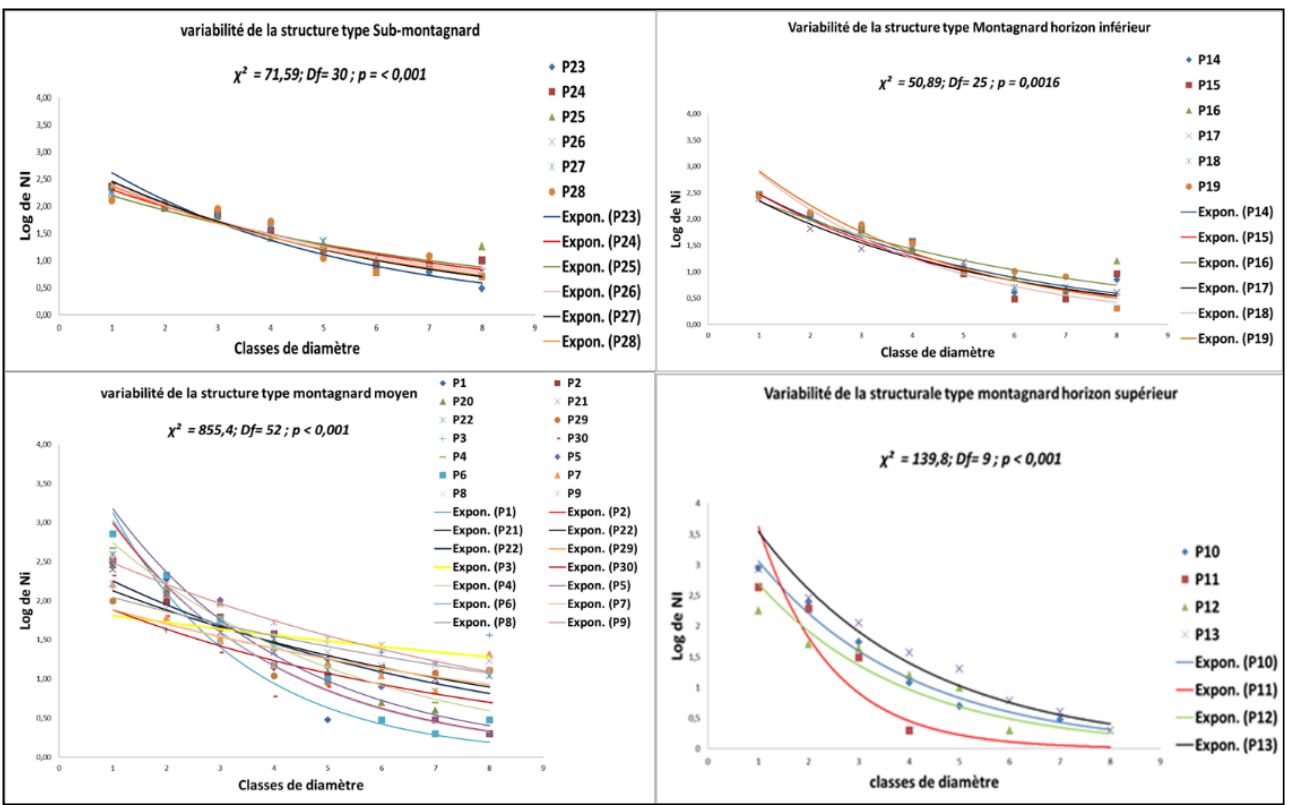

Figure 4: Distribution diamétrique des tiges inventoriées à l'intérieur de chaque type forestier. Le nombre d'individus est transformé en logarithme. 1= [ 10-20[, 2= [ 20-30[, 3= [ 30-40[, 4=[ 40-50[, 5=[ 50-60[, 6=[ 60-70[, 7=[ 70-80[, 8= 80. P1= Parcelle numéro 1,

P23= Parcelle numéro 23, etc. L'analyse de cette figure montre qu'au sein d'un type, certaines parcelles ont plus d'individus de petits diamètres qu'elles n'en ont de gros. Le diamètre moyen est de $27 \pm 17.5 \mathrm{~cm}$ pour l'altitude $1250-1500 \mathrm{~m}$, de $23 \pm 15 \mathrm{~cm}$ pour 1500 $1800 \mathrm{~m}$, de $24 \pm 18 \mathrm{~cm}$ entre $1800-2400 \mathrm{~m}$ et enfin de $18 \pm 9 \mathrm{~cm}$ entre $2400-2600 \mathrm{~m}$. le test de chi carré confirme la variabilité structurale pour chaque type forestier. Ainsi, par exemple, les parcelles 18 et 19 ont plus d'individus de petits diamètres que la parcelle 16 avec de gros diamètre pour le type montagnard horizon inférieur.

Les plus gros diamètres ont été trouvés dans les altitudes intermédiaires (entre 1800 et $2400 \mathrm{~m}$ ), au type montagnard horizon moyen pour les espèces : Chrysophyllum gorogosanum, Symphonia globulifera, Parinari excelsa, Carapa grandiflora, Cassipourea rwenzoriensis, Newtonia buchanani, Xymalos monospora. De même, pour le type Sub-montagnard, les gros diamètres sont observés chez Entadrophragma excelsa, Cynometra alexandrii, Leptonychia bampsii, Bakersideroxylon sp., Uapaca kirkiana et Celtis soyauxii. Au type montagnard horizon inférieur, ce sont les espèces Lebrunia buchaie, Piptadeniastrum africanum, Parinari excelsa, Syzygium guineense et Ocotea usambarensis enfin au type montagnard horizon supérieur chez Eucalyptus globulus, Agauria salicifolia, Myrica salicifolia et Hagenia abyssinica.

\section{Surface terrière et relation avec les autres paramètres structuraux}

La plus grande surface terrière est observée entre 1800-2400 m d'altitude au type montagnard moyen $\left(66,71 \mathrm{~m}^{2} / \mathrm{ha}\right)$ et la plus petite entre 
2400-2600 m au type forestier montagnard supérieur (14,18 $\left.\mathrm{m}^{2} / \mathrm{ha}\right)$. Pour l'écosystème étudié, la surface terrière moyenne est de $35,1 \pm 11,3 \mathrm{~m}^{2} / \mathrm{ha}$. D’une manière générale, la surface terrière n'est pas influencée par le gradient altitudinal $(D f=3, F=1.15$ et $p=0.35)$ figure 5 .

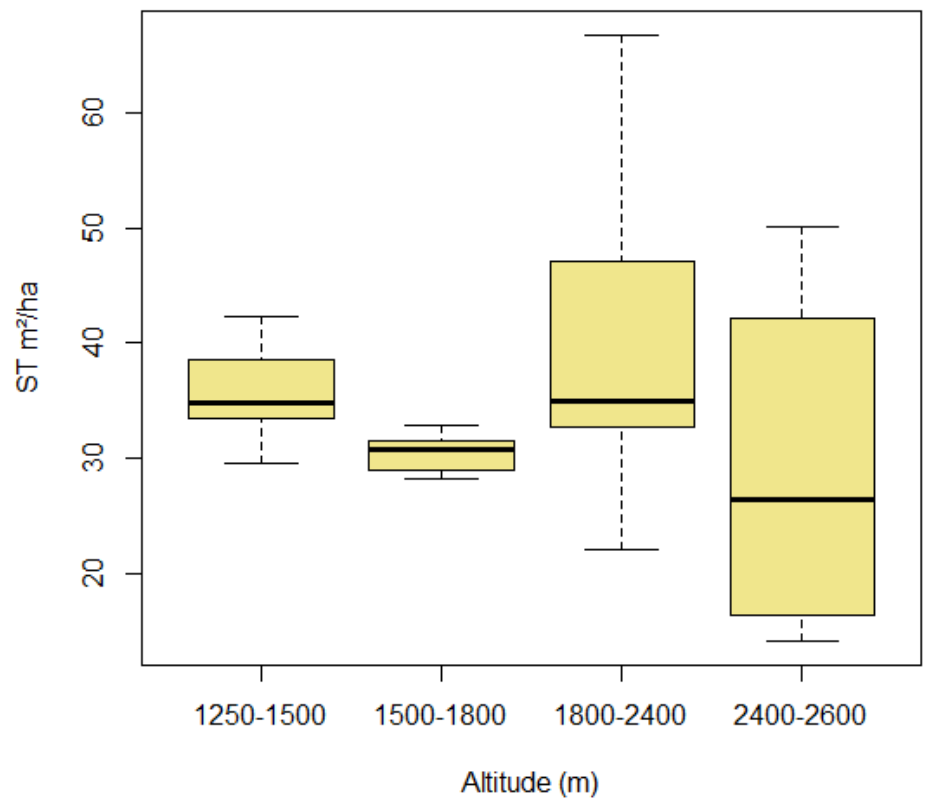

Figure 5 : Variation de la surface terrière entre les tranches d'altitude en forêt de montagne du PNKB et ses environs.

L'analyse de cette figure indique que la surface terrière varie fortement à l'horizon montagnard moyen (1800-2400) et supérieur (24002600). De même, les différences ne sont pas significatives entre surface terrière des types forestiers pris deux à deux (la différence Q de Tukey HSD variant entre - 1 et $8 ; p>0,05$ partout). Elle est 30,5 $\pm 1,7 \mathrm{~m}^{2} /$ ha entre 1250 $1500 \mathrm{~m} ; 35,6 \pm 4,4 \mathrm{~m}^{2} /$ ha entre $1500-1800 \mathrm{~m} ; 38,6 \pm 13,4 \mathrm{~m}^{2} /$ ha entre 1800 $2400 \mathrm{~m}$ et enfin de $29,3 \pm 16,3 \mathrm{~m}^{2} /$ ha entre $2400-2600 \mathrm{~m}$ d'altitude.

La surface terrière varie différemment avec les classes de diamètre le long du gradient altitudinal. La figure 6 indique que $21 \%$ de la surface terrière est réalisée par les deux premières classes (de 10 à $30 \mathrm{~cm}$ ) pour la tranche $1250-1500 \mathrm{~m}$; 31\% pour la tranche $1500-1800 \mathrm{~m}$; $26 \%$ entre 1800 $2400 \mathrm{~m}$ et enfin pour la tranche $2400-2600 \mathrm{~m}$, ces deux classes accumulent jusque $59 \%$ de la surface terrière totale. En considérant tous les types forestiers, il n'y a pas une relation entre la surface terrière et la densité ( $r$ $0,13 p$ value 0,49$)$ ni entre elle et le nombre d'espèce $(r-0,15 p$ value 0,40$)$. Aux altitudes supérieures, entre $1800-2400 \mathrm{~m}$ ( $r$ 0,87 p value <0,001) et $2400-2600 \mathrm{~m}$ ( $r$ 0,95 p value $<0,001)$, il existe une corrélation forte entre la surface terrière et la densité. 


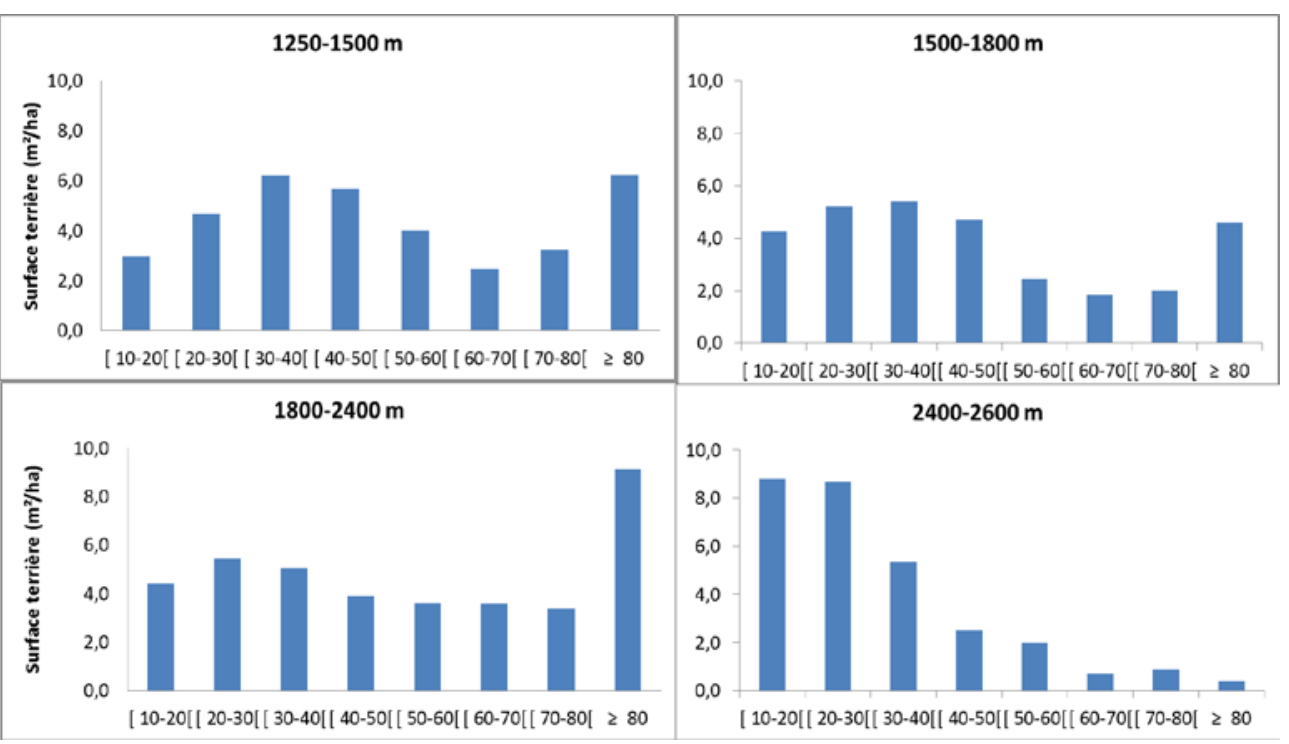

Figure 6: Distribution de la valeur de la surface terrière $\left(\mathrm{m}^{2} / \mathrm{ha}\right)$ le long du gradient altitudinal et suivant les classes de diamètre.

\section{Hauteur et relation avec d'autres paramètres structuraux}

La hauteur a été relevée sur 23 \% des individus inventoriés dans cette étude ; soit sur 3834 individus repartis sur l'ensemble des types forestiers et des classes de diamètre. En effet, elle varie suivant le gradient altitudinal ( $D f$ 3, $F$ value 61,5, $p$ value $<0,001$ ).

Le Système de classification IUFRO de la structure verticale distingue trois horizons. L'horizon supérieur avec les arbres d'une hauteur supérieure ou égale au 2/3 de la valeur maximale inventoriée; l'horizon moyen avec la hauteur des arbres comprise entre $1 / 3$ et $2 / 3$ de la valeur maximale et enfin l'horizon inférieur avec les arbres de la hauteur moins du $1 / 3$ de cette valeur maximale. La hauteur maximale de notre étude est de 48 $\mathrm{m}$ et notée à la tranche 1250-1500 m. Entre 1500-1800 m d'altitude la hauteur maximale est de $40 \mathrm{~m}$; de $35 \mathrm{~m}$ entre 1800-2400 m et enfin elle est de $29 \mathrm{~m}$ entre $2400-2600 \mathrm{~m}$.

L'abondance d'arbres n'est pas la même dans chaque horizon suivant les tranches d'altitude (tableau 3).

Tableau 3: Abondance d'arbres et leur pourcentage selon les tranches d'altitude

\begin{tabular}{cccccccccc}
\hline & \multicolumn{1}{c}{$1250-1500 \mathrm{~m}$} & $1500-1800 \mathrm{~m}$ & \multicolumn{2}{c}{$1800-2400 \mathrm{~m}$} & \multicolumn{2}{c}{$2400-2600 \mathrm{~m}$} \\
\cline { 2 - 9 } Classe de hauteur (m) & abond & $\%$ & abond & $\%$ & abond & $\%$ & abond & $\%$ \\
\hline Supérieure $\geq 32$ & 30 & 3,5 & 19 & 1,9 & 3 & 0,2 & 0 & 0 \\
Moyenne $16<\mathrm{H}<32$ & 254 & 29,4 & 261 & 26,7 & 294 & 18,0 & 5 & 1,4 \\
Inférieure $<16$ & 579 & 67,1 & 699 & 71,4 & 1334 & 81,8 & 356 & 98,6 \\
\hline
\end{tabular}

Légende $\mathrm{H}=$ Hauteur ; abond= abondance 
On note que très peu d’individus sont présent dans la classe supérieure constitutive de la voute de la forêt. Parmi les espèces qui la constituent, on peut citer Symphonia globulifera, Pycnanthus angolensis et Cynometra alexandrii pour la tranche 1250-1500 m ; Cynometra alexandrii, Bakersideroxylon sp., Lovoa trichilioides pour la tranche $1500-1800 \mathrm{~m}$ et enfin Piptadeniastrum africanum et Symphonia globulifera pour la tranche 1800-2400 m.

\section{Indices de Valeurs d'Importance (IVI) selon les tranches d'altitude}

Pour chaque type forestier, certaines espèces ont une importante valeur écologique sur base de la dominance relative (DOR) et de la densité relative (DR), en plus de leur nombre d'individus (NI). Le tableau 4 présente pour chaque tranche d'altitude, les dix premières espèces à indices de valeurs d'importance (IVI) plus important.

Tableau 4: Importance Value Index pour les dix espèces a grand score suivant les tranches altitudinales

\begin{tabular}{|c|c|c|c|c|c|}
\hline \multirow{2}{*}{ Familles } & \multirow{2}{*}{ Espèces } & \multicolumn{4}{|c|}{ Tranches d'altitude } \\
\hline & & $1250-1500$ & $1500-1800$ & $1800-2400$ & $2400-2600$ \\
\hline Ericaceae & Agauria salicifolia & & & & 24,4 \\
\hline Cornaceae & Alangium chinensis & & & 5,1 & \\
\hline Sapindaceae & Allophyllus kiwuensis & & & 6 & \\
\hline Annonaceae & Anonidium mannii & 24,5 & & & \\
\hline Meliaceae & Carapa grandiflora & & 28,9 & & \\
\hline Sapotaceae & Chrysophyllum gorungosanum & & & 12,8 & \\
\hline Putranjivaceae & Drypetes dinklagei & & 16,1 & & \\
\hline Moraceae & Ficus urceolaris & 5,1 & & & \\
\hline Clusiaceae & Garcinia punctata & & 6,6 & & \\
\hline Malvaceae & Grewia mildbraedii & & 11 & & \\
\hline Rosaceae & Hagenia abyssinica & & & & 43,7 \\
\hline Meliaceae & Leplea mayumbensis & & 7,1 & & \\
\hline Euphorbiaceae & Macaranga neomilbraediana & & & 70,8 & 32,8 \\
\hline Bignonaceae & Markhamia lutea & 9,3 & & & \\
\hline Moraceae & Myrianthus holstii & 5,9 & 5,6 & & \\
\hline Myricaceae & Myrica salicifolia & & & & 4,6 \\
\hline Stilbaceae & Nuxia congesta & & & & 2,3 \\
\hline Stilbaceae & Nuxia floribunda & & & & 32 \\
\hline Sapindaceae & Pancovia harmsiana & & 6 & & \\
\hline Apocynaceae & Pleiocarpa pycnantha & & & 7,2 & \\
\hline Araliaceae & Polyscias fulva & & & & 2,6 \\
\hline Myristicaceae & Pycnanthus angolensis & 14,1 & & & \\
\hline Myrsinaceae & Rapanea melanophloeos & & & & 36,8 \\
\hline Euphorbiaceae & Sapium ellipticum & & & 14,5 & \\
\hline Olacaceae & Strombosia grandiflora & & & 6,7 & \\
\hline Olacaceae & Strombosia scheffleri & 22,1 & & & \\
\hline Myrtaceae & Syzygium guineense & & & 10,2 & \\
\hline Euphorbiaceae & Tetrorchidium didymostemon & & 8,6 & & \\
\hline Meliaceae & Trichilia welwichii & 19,1 & & & \\
\hline Moraceae & Trilepisium madagascariensis & 11,9 & 31,8 & & \\
\hline Euphorbiaceae & Uapaca kirkiana & 5,2 & & & \\
\hline Monimiaceae & Xymalos monospora & 7,6 & 7,7 & 10,7 & 7,2 \\
\hline Ebenaceae & Diospyros Polystemone & & & 4,1 & \\
\hline Rubiaceae & Oxyanthus speciosus & & & & 2,1 \\
\hline
\end{tabular}


De part, leurs valeurs en IVI, Macaranga neomilbraediana est dominante dans les tranches 1800-2600 m d'altitude. Aussi Myrianthus holstii et Trilepisium madagascariensis entre 1250-1800 m. On remarque que Xymalos monospora domine sur l'ensemble de l'écosystème montagneux étudié surtout dans le sous-bois.

\section{Discussion}

\section{Variabilité de la structure diamétrique le long du gradient altitudinal}

La structure de la végétation et sa variabilité sont des indicateurs du fonctionnement de l'écosystème et de son état (Ostertag et al., 2014). Il est démontré que le patron de la composition structurale et de la distribution des communautés végétales forestières en fonction de l'altitude dépendent des caractéristiques écologiques de la végétation (Givnish, 1999; Lovett et al., 2006; Alves et al., 2010; Biresaw and Pavliš, 2010; Mangambu et al., 2015). Il ne s’agit pas simplement de lister une série d’espèces végétales, mais d'explorer les différents mécanismes qui permettent leur coexistence (Mangambu, 2013). Dans cette étude, on a remarqué que l'allure de la courbe individus diamètre est en $\mathrm{J}$ inversé typique de forêt tropicale en dynamique progressive (Hitimana et al., 2004; Pascal, 2006). Les classes inférieures (de 10 à $30 \mathrm{~cm}$ ) ont plus d’individus que les classes supérieures mais cela varie selon le gradient altitudinal. Par exemple, entre 1250-1500 m, on note que $66 \%$ d'individus se retrouvent entre les classes de diamètre de 10 à 30 cm, 76 \% d'individus pour la tranche 1500-1800 m d'altitude tandis qu'on retrouve $80 \%$ d'individus entre 1800-2400 m et $90 \%$ d'individus pour la tranche $2400-2600 \mathrm{~m}$. Cette diminution de la taille des arbres s'expliquerait par l'adaptation des espèces à la diminution de la température et variations d'autres facteurs en altitude (Pierlot, 1966) surtout en zone de montagne. Mangambu et al., (2015) admettent que les formations forestières montagnardes présentent différentes structurations floristiques d’après les tranches d'altitude, leur diversité floristique et potentiellement leur fonctionnement dans le PNKB.

C'est dans ce sens que la tranche d'altitude 1800-2400 m présente des particularités ; Symphonia globulifera qui a le plus gros diamètre de tous les inventaires se retrouve dans la parcelle de cette tranche qui a également un nombre important d'individus de petits diamètres (soit $80 \%$ ). Ainsi, selon qu'il s'agisse d'une formation primaire ou secondaire la structure de la végétation pourrait changer (Lieberman et al., 1996; Zapfack et al., 2002; Bouko et al., 2007; Rahaingoson et al., 2013; Lee et al., 2014).

La variabilité structurale pourrait être différente d'un site à un autre selon les conditions écologiques stationnelles (Lovett et al., 2006). Dans le contexte d'une haute altitude, par exemple, Masumbuko et al., (2012) ont remarqué que l'invasion de la forêt par la liane Sericostachys scandens 
depuis 2007 avait fortement modifié la structure de la forêt entre 1800 et $2400 \mathrm{~m}$ d'altitude en faisant tomber les arbres. Le changement dans les facteurs climatiques a influencé, dans le passé, la structure et la composition de la végétation au PNKB et ses environs (Kabonyi et al., 2011). Les espèces ont migré vers les altitudes supérieures pour s’adapter à l'augmentation de la température dans la zone. Au cours de 40 dernières années passées, les recherches confirment une augmentation de la température et une perturbation du régime hydrique dans la région (Akonkwa et al., 2015). Bien qu'aucun lien ne soit fait actuellement avec la végétation dans la région, il s'avère important d'analyser l'impact du changement climatique sur les écosystèmes forestiers. C’est dans ce cas que Imani et al., (2016b) déterminent que le climat pourrait être le facteur qui influence la structuration du peuplement de Hagenia abyssinica dans la haute altitude du PNKB.

\section{Changement dans la surface terrière en relation avec le diamètre et la densité des arbres le long du gradient altitudinal}

La surface terrière et la densité sont deux attributs structurels qui renseignent sur la couverture de la forêt et son état (Bogaert et Mahamane, 2005). Comme la densité, la surface terrière varie le long du gradient altitudinal. En moyenne, la surface terrière est de 35,12 $\mathrm{m}^{2} / \mathrm{ha}$. La plus grande surface est observée entre $1800-2400 \mathrm{~m}$ d'altitude $\left(66,71 \mathrm{~m}^{2} / \mathrm{ha}\right)$ et la plus petite entre $2400-2600 \mathrm{~m}\left(14,18 \mathrm{~m}^{2} / \mathrm{ha}\right)$. Les résultats ont montré que la surface terrière ne diminue pas statistiquement lorsqu'on monte en altitude et qu'il n'existe pas de différence entre les tranches d'altitude prises deux à deux. Par ailleurs, on a observé une forte variabilité de la surface terrière pour les altitudes supérieures (soit 38,6 $\pm 13,4 \mathrm{~m}^{2} /$ ha entre $1800-2400 \mathrm{~m}$ et $29,3 \pm 16,3 \mathrm{~m}^{2} /$ ha entre $2400-2600 \mathrm{~m}$ d'altitude). Toutefois, la faible valeur en surface terrière dans les altitudes supérieures pourrait s'expliquer par le changement observé dans la taille des arbres et la composition floristique. Par ailleurs, cette absence de la différence significative de surface terrière pourrait aussi résulter des prélèvements opérés par les communautés locales sur la ressource. Prélèvements plus importants sur les zones les plus basses. Ce qui corroborerait une diminution de surface terrière observable mais pas significative.

Pour l'altitude de 2400 à $2600 \mathrm{~m}$, on note une relation positive surface terrière et nombre d'individus ainsi que la diminution en diamètre des arbres pour s'adapter aux conditions climatiques de montagne (Toledo et al., 2012). Ainsi, la valeur inférieure de la surface terrière est retrouvée pour la parcelle avec une densité faible (301 individus) et de faible diamètre (78 cm maximum) alors que la valeur élevée est notée pour la parcelle de cette série avec la densité moyenne (361 individus) mais des gros arbres (200 cm 
maximum). Ceci suggère que le recouvrement forestier dans cette zone de montagne varie à la fois avec la densité, le diamètre et la composition floristique. Par exemple, pour la tranche 2400-2600 m, 90 \% d'individus se trouvent entre 10-30 cm et accumulent jusqu’à $59 \%$ de la surface terrière. Ainsi, la densité des arbres et la composition influencent la surface terrière en altitude supérieure (au-delà de $2400 \mathrm{~m}$ d’altitude) alors que le diamètre des arbres est déterminant pour la surface terrière en dessous de $2400 \mathrm{~m}$ d'altitude.

Dans les montagnes du Kivu, Pierlot, (1966) parle d'une surface terrière variant entre 18 et $37 \mathrm{~m}^{2}$ et précise par contre que la surface terrière (ST) et le nombre d'individus (NI) sont généralement importants en altitude lorsque la forêt n’est pas perturbée. Dans le Rift albertin, Eilu et al., (2004), notent une surface terrière de 14,5 à $45 \mathrm{~m}^{2} /$ ha en Ouganda, alors que Lovett et al., (2006) précisent que la valeur de la surface terrière peut aller jusqu’à $70 \mathrm{~m}^{2} /$ ha en Tanzanie selon les formations végétales tout en précisant que la relation densité et surface terrière est positive. Pour les montagnes de l'Inde, Sahu et al., (2008) déterminent la surface terrière variant entre 7 et $74 \mathrm{~m}^{2} / \mathrm{ha}$ et qui augmente avec l'altitude mais pas avec la densité. Dans les montagnes en Ethiopie, Gurmessa et al., (2012) trouvent une surface terrière de $51 \mathrm{~m}^{2} / \mathrm{ha}$ et précisent que les classes de diamètre de 10 à $30 \mathrm{~cm}$ contribuent faiblement dans la valeur de la surface terrière. De ces faits, la structure de la végétation en zone de montagne varie selon chaque région ou site (Bussmann, 2006).

\section{Variation de la structure verticale le long du gradient altitudinal}

La hauteur des arbres en relation avec le diamètre varie selon les sites et les régions (Feldpausch et al., 2011) en zone tropicale. On a trouvé la hauteur la plus élevée d'environ 48 m entre 1250-1500 m d'altitude alors que la hauteur maximale était de $29 \mathrm{~m}$ entre 2400-2600 m. Selon les classes de hauteur, 99 \% d'individus inventorié entre 2400-2600 m d'altitude et $82 \%$ entre 1800-2400 m ont une valeur de la hauteur inférieure à 16 m contre 66 $\%$ entre $1250-1500 \mathrm{~m}$ et $71 \%$ entre $1500-1800 \mathrm{~m}$. Par ailleurs, on a remarqué une corrélation positive entre la hauteur et le diamètre comme dans beaucoup d'autres études (Feldpausch et al., 2011, 2012). Ceci montre que l'altitude influence la structure verticale de la végétation en forêt de montagne au PNKB et ses environs et que cette variabilité est importante lorsque les altitudes deviennent très élevées. Dans les mêmes montagnes du Rift albertin en Tanzanie, on a inventorié une hauteur maximale de $45 \mathrm{~m}$ (Lovett et al., 2006) similaire à la valeur inventoriée dans cette étude.

Les études ont démontré que la hauteur est un bon facteur pour améliorer la précision dans l'estimation de la biomasse en zone tropicale (Lewis et al., 2009; Feldpausch et al., 2012; Hunter et al., 2013). Les résultats montrent une variation altitudinale de la hauteur et du diamètre des 
arbres. Ce qui suggère une nécessité d’élaborer les modèles de relation hauteur diamètre spécifique à chaque type forestier le long du gradient altitudinal et de les comparer aux modèles régionaux existants (Feldpausch et al., 2012).

\section{Dominance des espèces et des familles}

La dominance des espèces dans chaque type forestier a été déterminée sur base de leur pourcentage en IVI (Tableau 4). Les dix premières espèces ont été considérées dominantes à cause de leur abondance et grande surface terrière dans le massif forestier. Ces espèces dominantes indiquent par leur tempérament par rapport à la lumière l'état de l'écosystème, selon qu'elles sont sciaphiles, héliophile, hemisciaphiles ou xérohéliophytes (Ngueguim, 2013). Le tableau 5 synthétise le tempérament des espèces dominantes inventoriées selon Pierlot, (1966) :

Tableau 5: Espèces dominantes et leur tempérament selon les tranches d'altitude

\begin{tabular}{|c|c|c|c|c|}
\hline Tranche & Sciaphiles & Hemisciaphiles & Héliophyles & Xérohéliophyles \\
\hline \multirow{3}{*}{$\begin{array}{c}1250- \\
1800\end{array}$} & \multirow{3}{*}{$\begin{array}{c}\text { Drypetes } \\
\text { dinklagei } \\
\text { Anonidium } \\
\text { mannii } \\
\text { Trichilia } \\
\text { welwichii }\end{array}$} & Strombosia scheffleri & $\begin{array}{l}\text { Pycnanthus } \\
\text { angolensis }\end{array}$ & \\
\hline & & Carapa grandiflora & Markhamia lutea & \\
\hline & & Grewia mildbraedii & $\begin{array}{c}\text { Trilepisium } \\
\text { madagascariensis } \\
\text { Tetrorchidium } \\
\text { didymostemon }\end{array}$ & \\
\hline \multirow{3}{*}{$\begin{array}{l}1800- \\
2600\end{array}$} & $\begin{array}{c}\text { Xymalos } \\
\text { monospora }\end{array}$ & $\begin{array}{c}\text { Chrysophyllum } \\
\text { gorogosanum }\end{array}$ & $\begin{array}{c}\text { Macaranga } \\
\text { neomilbraediana }\end{array}$ & \multirow{3}{*}{$\begin{array}{c}\text { Rapanea } \\
\text { melanophloeos } \\
\text { Nuxia floribunda } \\
\text { Agauria } \\
\text { salicifolia }\end{array}$} \\
\hline & Syzy & um guineense & Sapium ellipticum & \\
\hline & & Strombosia scheffleri & Hagenia abyssinica & \\
\hline
\end{tabular}

Les espèces Sciaphiles et Hémisciaphiles sont dominantes dans les formations matures ou dans le sous-bois (Xymalos monospora) alors que les Héliophyles sont des formations secondaires et les Xérophiles spécifiques aux altitudes supérieures à $2400 \mathrm{~m}$.

Dans le cas du Rift albertin, Eilu et al., (2004) trouvent que les familles abondantes pour les ligneux sont Euphorbiaceae, Meliaceae, Rubiaceae, Fabaceae et Sapotaceae dans les forêts de montagne en Ouganda. On retrouve plusieurs de ces familles dans notre cas, bien que les abondances varient selon les tranches. Globalement, il s'agit des Euphorbiaceae, Moraceae, Meliaceae, Fabaceae et Rubiaceae.

\section{Conclusion}

La variabilité structurale des espèces ligneuses à $\mathrm{dbh} \geq 10 \mathrm{~cm}$ a été analysée dans ce travail le long d'un gradient altitudinal pour les formations 
de montagne situées entre 1250 et 2600 m d'altitude au PNKB et ses environs. L’objectif de l'étude a été de déterminer le changement de la structure diamétrique, de la densité, de la surface terrière et de la hauteur des arbres selon les tranches d'altitude ou types forestiers (sub-montagnard 1250-1500 m, montagnard horizon inférieur 1500-1800 m; montagnard horizon moyen 1800-2400 m et montagnard horizon supérieur 2400-2600 m) tel qu'ils sont distingués en forêt de montagne de cette zone de la région du Rift albertin.

Les nombres de famille, de genre et d'espèce régressent lorsque l'altitude augmente pendant que la densité croit proportionnellement au gradient altitudinal; on note un nombre élevé d'individus aux altitudes supérieures entre 2400-2600 m.

La structure diamétrique de l'ensemble du massif montagneux en J inversé, montre que les formations forestières suivent une dynamique progressive avec beaucoup d'individus dans les premières classes de diamètre de $10-30 \mathrm{~cm}$. Cette structure présente de la variation à chaque tranche d'altitude due notamment aux conditions locales, à l'état de la végétation, à l’intensité de la pression humaine ou naturelle.

La surface terrière moyenne est de $35 \mathrm{~m}^{2} /$ ha et varie entre 14 à 67 $\mathrm{m}^{2} / \mathrm{ha}$. On n’a pas déterminé la relation entre l'altitude et la surface terrière en général ni entre la surface terrière et les types forestiers comparés deux à deux. Tout de même la faible surface terrière est observée aux altitudes supérieures entre 2400-2600 m en raison de la taille réduite des arbres et la composition floristique en altitude. Le nombre d'individus est un facteur déterminant pour la surface terrière en altitude pendant que le diamètre des arbres et la densité sont importants pour un meilleur recouvrement forestier aux altitudes inférieures à $2300 \mathrm{~m}$.

La structure verticale en zone de montagne au PNKB et ses environs est influencée par le gradient altitudinal. La hauteur moyenne est de $48 \mathrm{~m}$. Elle devient faible en altitude supérieure pendant que la densité augmente. La relation hauteur diamètre varie selon les tranches d'altitude suivant la densité des arbres et la composition floristique locale.

Les espèces dominantes sont différentes selon les tranches d'altitude. Les espèces Strombosia scheffleri, S. grandiflora et Xymalos monospora (dans le sous-bois) dominent sur l'ensemble de la forêt étudiée. Ce qui confirme l'appartenance de ces forêts à la classe phytosociologique « Strombosio-parietea ».

Les forêts de montagne au PNKB et ses environs sont des formations à Meliaceae, Moraceae, Euphorbiaceae, Fabaceae et Rubiaceae.

En conclusion, la variabilité structurale de la végétation des écosystèmes montagneux au PNKB et ses environs est évidente et devient de plus en plus forte lorsque les altitudes s’éloignent. Cette variabilité devait 
être prise en compte lorsqu'on cherche à comprendre le fonctionnement de ces écosystèmes notamment leur rôle dans l'accumulation de la biomasse aérienne ligneuse pour atténuer les effets négatifs du changement climatique. Mais également dans le but d'améliorer leur système de gestion et d'aménagement.

Le changement climatique influence la structure et la composition de la végétation. Dans le contexte du PNKB et ses environs, l'évolution probable de la structure floristique et la migration des espèces végétales sur le gradient altitudinal peut influencer la fonction de stockage de carbone et modifier ainsi le rôle de ces écosystèmes montagneux dans la lutte contre le changement climatique. Il s'avère important d'analyser ces aspects de changement climatique pour améliorer la politique de gestion et d'aménagement des écosystèmes forestiers montagnards.

\section{Remerciements}

Le présent travail a été soutenu financièrement par le projet FCCC (Appui à l'UNIKIS: "Forêts et Changement Climatique au Congo") financé par la Commission européenne, mis en œuvre par le CIFOR en partenariat avec l'Université de Kisangani (UNIKIS) et RSD pour le soutien logistique. Les auteurs remercient également tous ceux qui ont contribué à la collecte et au traitement des données.

\section{References:}

Akonkwa, B., Muhigwa, B., Montcho, S.A., Laleye, P. (2015). Climate change and its impact on the fisheries in Lake Kivu , East Africa. Journal of Biodiversity and Ecological Sciences, 6, 312-327.

Alves, L.F., Vieira, S.A., Scaranello, M.A., Camargo, P.B., Santos, F.A.M., Joly, C.A., Martinelli, L.A. (2010). Forest structure and live aboveground biomass variation along an elevational gradient of tropical Atlantic moist forest (Brazil). Forest Ecology and Management, 260, 679-691. doi:10.1016/j.foreco.2010.05.023

Baraloto, C., Molto, Q., Rabaud, S., Hérault, B., Valencia, R., Blanc, L., Fine, P.V. A., Thompson, J. (2013). Rapid Simultaneous Estimation of Aboveground Biomass and Tree Diversity Across Neotropical Forests: A Comparison of Field Inventory Methods. Biotropica, 45, 288-298. doi:10.1111/btp.12006

Bastin, J.-F., Barbier, N., Réjou-Méchain, M., Fayolle, A., Gourlet-Fleury, S., Maniatis, D., de Haulleville, T., Baya, F., Beeckman, H., Beina, D., Couteron, P., Chuyong, G., Dauby, G., Doucet, J.-L., Droissart, V., Dufrêne, M., Ewango, C., Gillet, J.F., Gonmadje, C.H., Hart, T., Kavali, T., Kenfack, D., Libalah, M., Malhi, Y., Makana, J.-R., Pélissier, R., Ploton, P., Serckx, A., Sonké, B., Stevart, T., Thomas, D.W., De Cannière, C., Bogaert, J. 
(2015). Seeing Central African forests through their largest trees. Sci. Rep., 5, 1-8. doi:10.1038/srep13156

Biresaw, M. A., Pavliš, J. (2010). Vegetation structure and density of woody plant species in two woodland areas of Amhara National Regional State, Ethiopia. Acta Univ. Agric. Silvic. Mendelianae Brun., 58, 21-32.

Bogaert, J., Mahamane, A. (2005). Ecologie du paysage: cibler la configuration et l'échelle spatiale. Ann. des Sci. Agron. du Benin, 7, 39-68.

Bouko, B.S., Sinsin, B., Soulé, B.G. (2007). Effets de la dynamique d'occupation du sol sur la structure et la diversité floristique des forêts claires et savanes au Bénin. Tropicultura, 25, 221-227.

Boyemba, F. (2011). Ecologie de Pericopsis elata (Harms) Van Meeuwen (Fabaceae), arbre de forêt tropicale africaine à répartition agrégée. Thèse de Doctorat, Université Libre de Bruxelles, 206p.

Curtis, J.., Mclntosh, R.(1950). The interrelations of certain analytic and synthetic phytosociological characters. Ecology, 31, 434-455. doi:10.2307/1931497

Dale, V.H., Joyce, L.A., McNulty, S., Neilson, R.P. (2000). The interplay between climate change, forest, and disturbances. Sci. Total Environ., 262, 201-204.

Eilu, G., Hafashimana, D.L.N., Kasenene, J.M. (2004). Density and species diversity of trees in four tropical forests of the Albertine rift, western Uganda. Divers. Distrib., 10, 303-312. doi:10.1111/j.13669516.2004.00089.X

Fashing, P.J., Gathua, J.M., 2004. Spatial variability in the vegetation structure and composition of an East African rain forest. Afr. J. Ecol., 42, 189-197.

Feldpausch, T.R., Banin, L., Phillips, O.L., Baker, T.R., Lewis, S.L., Quesada, C.A., Affum-Baffoe, K., Arets, E.J.M.M., Berry, N.J., Bird, M., Brondizio, E.S., de Camargo, P., Chave, J., Djagbletey, G., Domingues, T.F., Drescher, M., Fearnside, P.M., França, M.B., Fyllas, N.M., Lopez-Gonzalez, G., Hladik, A., Higuchi, N., Hunter, M.O., Iida, Y., Salim, K.A., Kassim, A.R., Keller, M., Kemp, J., King, D.A., Lovett, J.C., Marimon, B.S., Marimon-Junior, B.H., Lenza, E., Marshall, A.R., Metcalfe, D.J., Mitchard, E.T.A., Moran, E.F., Nelson, B.W., Nilus, R., Nogueira, E.M., Palace, M., Patiño, S., Peh, K.S.-H., Raventos, M.T., Reitsma, J.M., Saiz, G., Schrodt, F., Sonké, B., Taedoumg, H.E., Tan, S., White, L., Wöll, H., Lloyd, J. (2011). Height-diameter allometry of tropical forest trees. Biogeosciences, 8, 1081-1106. doi:10.5194/bg-8-1081-2011

Feldpausch, T.R., Lloyd, J., Lewis, S.L., Brienen, R.J.W., Gloor, M., Monteagudo Mendoza, A., Lopez-Gonzalez, G., Banin, L., Abu Salim, K., Affum-Baffoe, K., Alexiades, M., Almeida, S., Amaral, I., Andrade, A., Aragão, L.E.O.C., Araujo Murakami, A., Arets, E.J.M.M., Arroyo, L., 
Aymard C., G.A., Baker, T.R., Bánki, O.S., Berry, N.J., Cardozo, N., Chave, J., Comiskey, J.A., Alvarez, E., de Oliveira, A., Di Fiore, A., Djagbletey, G., Domingues, T.F., Erwin, T.L., Fearnside, P.M., França, M.B., Freitas, M.A., Higuchi, N., E. Honorio C., Iida, Y., Jiménez, E., Kassim, A.R., Killeen, T.J., Laurance, W.F., Lovett, J.C., Malhi, Y., Marimon, B.S., MarimonJunior, B.H., Lenza, E., Marshall, A.R., Mendoza, C., Metcalfe, D.J., Mitchard, E.T.A., Neill, D.A., Nelson, B.W., Nilus, R., Nogueira, E.M., Parada, A., Peh, K.S.-H., Pena Cruz, A., Peñuela, M.C., Pitman, N.C.A., Prieto, A., Quesada, C.A., Ramírez, F., Ramírez-Angulo, H., Reitsma, J.M., Rudas, A., Saiz, G., Salomão, R.P., Schwarz, M., Silva, N., Silva-Espejo, J.E., Silveira, M., Sonké, B., Stropp, J., Taedoumg, H.E., Tan, S., ter Steege, H., Terborgh, J., Torello-Raventos, M., van der Heijden, G.M.F., Vásquez, R., Vilanova, E., Vos, V.A., White, L., Willcock, S., Woell, H., Phillips, O.L. (2012). Tree height integrated into pantropical forest biomass estimates. Biogeosciences, 9, 3381-3403. doi:10.5194/bg-9-3381-2012

Givnish, T.J., 1999. On the causes of gradients in tropical tree species diversity. J. Ecol., 87, 193-210.

Gurmessa, F., Soromessa, T., Kelbessa, E. (2012). Structure and regeneration status of Komto Afromontane moist forest, East Wollega Zone, west Ethiopia. J. For. Res., 23, 205-216. doi:10.1007/s11676-012-0242-8

Hitimana, J., Kiyiapi, J.L., Njunge, J.T. (2004). Forest structure characteristics in disturbed and undisturbed sites of Mt. Elgon Moist Lower Montane Forest, western Kenya. For. Ecol. Manage., 194, 269-291. doi:10.1016/j.foreco.2004.02.025

Huang, W.D., Pohjonen, V., Johansson, S., Nashanda, M., Katigula, M.I.L., Luukkanen, O. (2003). Species diversity, forest structure and species composition in Tanzanian tropical forests. For. Ecol. Manage., 173, 11-24. doi:10.1016/s0378-1127(01)00820-9

Hunter, M.O., Keller, M., Victoria, D., Morton, D.C. (2013). Tree height and tropical forest biomass estimation. Biogeosciences, 10, 8385-8399. doi:10.5194/bg-10-8385-2013.

Imani, M. G., Zapfack, L., Kalume, J., Riera, B., Cirimwami, L., and Boyemba, F. (2016a). Woody vegetation groups and diversity along the altitudinal gradient in mountain forest : case study of Kahuzi-Biega National Park and its surroundings, RD Congo. Journal of Biodiversity and Environmental Sciences, 8:134-150.

Imani, M., Zapfack, L., Mangambu, M., Penedimanja, P., Mwanga Mwanga, J.I., Boyemba, F. (2016b). Caractérisations Structurale, Floristique Et Biomasse Ligneuse Du Peuplement À Hagenia abyssinica Du Parc National De Kahuzi-Biega (Rift Albertin, RDC). Eur. Sci. J., 12, 189-209. doi:10.19044/esj.2016.v12n15p189

Kabonyi, N.C. (2012). Analyse palynologique de divers sondages du secteur 
de haute altitude du Parc National de Kahuzi-Biega. Paléoenvironnements et paléoclimats au Pléistocène supérieur et à l'Holocène. Thèse de Doctorat, Université de Liège, 128p.

Kabonyi, Z., Salmon, M., Roche, E., 2011. Le Parc National de Kahuzi Biega (RD. Congo), patrimoine en péril ? Le secteur «Haute Altitude », situation et perspectives . Geo-Eco-Trop., 34, 1-8.

Lasch, P., Lindner, M., Erhard, M., Suckow, F., Wenzel, A. (2002). Regional impact assessment on forest structure and functions under climate change-the Brandenburg case study. For. Ecol. Manage., 162, 73-86. doi:10.1016/S0378-1127(02)00051-8

Lee, C.B., Chun, J.H., Ahn, H.H. (2014). Elevational patterns of plant richness and their drivers on an Asian mountain. Nord. J. Bot., 32, 347-357. doi:10.1111/j.1756-1051.2013.00181.x

Lewis, S.L., Lopez-Gonzalez, G., Sonké, B., Affum-Baffoe, K., Baker, T.R., Ojo, L.O., Phillips, O.L., Reitsma, J.M., White, L., Comiskey, J. a, Djuikouo K, M.-N., Ewango, C.E.N., Feldpausch, T.R., Hamilton, A.C., Gloor, M., Hart, T., Hladik, A., Lloyd, J., Lovett, J.C., Makana, J.-R., Malhi, Y., Mbago, F.M., Ndangalasi, H.J., Peacock, J., Peh, K.S.-H., Sheil, D., Sunderland, T., Swaine, M.D., Taplin, J., Taylor, D., Thomas, S.C., Votere, R., Wöll, H. (2009). Increasing carbon storage in intact African tropical forests. Nature, 457, 1003-1006. doi:10.1038/nature07771

Lieberman, D., Lieberman, M., Peralta, R., Hartshorn, G. (1996). Tropical forest structure and composition on a large scale altitudinal gradient in Costa Rica. J. Ecol., 84, 137-152.

Lovett, J.C., Marshall, A.R., Carr, J. (2006). Changes in tropical forest vegetation along an altitudinal gradient in the Udzungwa Mountains National Park, Tanzania. Afr. J. Ecol., 44, 478-490. doi:10.1111/j.13652028.2006.00660.x

Mangambu, J. (2013). Taxonomie, biogéographie et écologie des Ptéridophyes de l'écosystème forestier des montagnes du Parc National de Kahuzi-Biega à l'Est de la R.D.Congo. Thèse de Doctorat, Université d'Anvers, 494p.

Mangambu, J.D.D.M., Muhashy, F., Robbrecht, E., Janssen, T., Ntahobavuka, H.H., Ruud, D. (2015). Ptéridophytes: Bio indicateurs des changements opérés sur la structure des forêts de zone de montagnes du Parc National de Kahuzi-Biega à l'Est de la R.D. Congo. Int. J. Innov. Sci. Res., 16, 350-370.

Mangambu, M.J., Habiyaremye, M., Janssen, T., Van Diggelen, R., Robbrecht, E., Ntahobavuka, H.H. (2013). Diversité des Fougères et leurs alliées le long du gradient altitudinal au sein de l'écosystème forestier des montagnes du Parc National de Kahuzi- Biega (RD. Congo ). Int. J. Environnemental Stud., 70, 259-283. 
Mangambu, M.J.D., Noiha Noumi, V., Zapfack, L., Sonké, E.B. (2010). Etude phytosociologique du groupement à Piper capensis (RD. Congo). Int. J. Environ. Stud., 67, 417-430. doi:10.1080/00207231003694033

Masumbuko, N., Habiyaremye, M., Lejoly, J. (2012). Woody climbing plants influence the structure of the mountain forest in the Kahuzi-Biega National Park , DR Congo. Reg. environnemental Chang., 12, 951-959. doi:10.1007/s10113-012-0309-2

Ngueguim, J.R. (2013). Productivité et diversité floristique des ligneux en forêt dense d'Afrique tropicale humide du Cameroun: Sites de Mangombe Bidou. Thèe de Doctorat, Museum National d'Histoire Naturelle, 213p.

Ostertag, R., Inman-Narahari, F., Cordell, S., Giardina, C.P., Sack, L. (2014). Forest structure in low-diversity tropical forests: a study of Hawaiian wet and dry forests. PLoS One, 9, 1-18. doi:10.1371/journal.pone.0103268

Pascal, J. (2003). Notions sur les structures et dynamique des forêts tropicales humides. Revue Forestière Française, numéro special 2003, 118130.

Phillips, O.L., Hall, P., Gentry, A.H., Sawyer, S.A, Vásquez, R. (1994). Dynamics and species richness of tropical rain forests. Proc. Natl. Acad. Sci. U.S.A., 91, 2805-2809. doi:10.1073/pnas.91.7.2805

Pierlot, R. (1966). Structure et composition de forest denses d'Afrique Centrale, specialement celles du Kivu, Académie Royale des sciences d'Outre mer, Editions Bruxelles, 373p.

Proctor, J., Anderson, J.M., Chai, P., Vallack, H.W.(1983). Ecological studies in four contrasting Lowland Rain forests in Gunung Mulu National Park, Sarawak: I. Forest Environment, Struture and Floristics. J. Ecol., 71, 237-260.

Rahaingoson, F., Rakotoarimanana, V., Roger, E. (2013). Analyse structurale et floristique de la végétation selon les différents types de gestion sur le Plateau Calcaire Mahafaly, published in: Rôle et place des transferts de gestion des ressources naturelles renouvelables dans les politiques forestières actuelles à Madagascar, Madagascar (2013), 8p.

Ren, H.B., Niu, S.K., Zhang, L.Y., Ma, K.P. (2006). Distribution of vascular plant species richness along an elevational gradient in the Dongling Mountains, Beijing, China. J. Integr. Plant Biol., 48, 153-160. doi:10.1111/j.1744-7909.2006.00153.x

Runge, J. (2007). Des déserts et des forêts , histoire du paysage et du climat de l'Afrique Centrale au Quaternaire Supérieur. Geo-Eco-Trop., 31, 1-18.

Sahu, P.K., Sagar, R., Singh, J.S. (2008). Tropical forest structure and diversity in relation to altitude and disturbance in a Biosphere Reserve in central India. Appl. Veg. Sci., 11, 461-470. doi:10.3170/2008-7-18537

Ter Steege, H., Sabatier, D., Castellanos, H., Van Andel, T., Duivenvoorden, J., Adalardo De Oliveira, A., Ek, R., Lilwah, R., Maas, P., Mori, S. (2000). 
An analysis of the floristic composition and diversity of Amazonian forests including those of the Guiana Shield. J. Trop. Ecol., 16, 801-828. doi:10.1017/S0266467400001735

Terborgh, J., Andresen, E. (1998). The composition of Amazonian forests: patterns at local and regional scales. J. Trop. Ecol., 14, 645-664. doi:10.1017/S0266467498000455

Wagner, F., Rutishauser, E., Blanc, L., Herault, B. (2010). Effects of Plot Size and Census Interval on Descriptors of Forest Structure and Dynamics. Biotropica, 42, 664-671. doi:10.1111/j.1744-7429.2010.00644.x

Werger, M.J.A. (1973). Notes on the phytogeographical affinities of the southern Kalahari. Bothalia, 11, 177-180.

Yamagiwa, J., Basabose, A.K., Kaleme, K., Yumoto, T. (2005). Diet of Grauer's Gorillas in the Montane Forest of Kahuzi, Democratic Republic of Congo. Int. J. Primatol., 26, 1345-1373.

Zapfack, L., Engwald, S., Sonke, B., Achoundong, G., Madong, B.A. (2002). The impact of land conversion on plant biodiversity in the forest zone of Cameroon. Biodivers. Conserv., 11, 2047-2061. 